\title{
Cortical Laminar Necrosis and Migrainous Stroke
}

\author{
Simona Lattanzi ${ }^{1} \quad$ Claudia Rinaldi ${ }^{1}$ Mauro Dobran ${ }^{2}$ \\ ${ }^{1}$ Department of Experimental and Clinical Medicine, Neurological \\ Clinic, Marche Polytechnic University, Ancona, Italy \\ 2Department of Experimental and Clinical Medicine, Clinic of \\ Neurosurgery, Marche Polytechnic University, Ancona, Italy
}

J Neurosci Rural Pract 2019;10:563-564

In the present article, authors report a case of cortical laminar necrosis (CLN) as the presenting neuroimaging sign of cerebral infarct in a 27-year-old woman with a history of migraine. ${ }^{1}$ This case report provides useful insights and reflections about major topics and challenges in the landscape of acute vascular neurology.

\section{Migrainous Stroke: An Intriguing and Still Not Completely Understood Entity}

Migraine is one of the most common neurological disorders with an estimated prevalence of around $15 \%$ and more than one billion people presenting this condition worldwide. Migraine is characterized by recurrent attacks of headache lasting 4 to 72 hours. The pain is generally unilateral, throbbing, moderate-to-severe in intensity, associated with nausea and/or vomiting, photo- and/or phonophobia, and worsened by physical activity. About one-third of migraineurs also experience aura symptoms, most often of visual type.

Although migraine is considered a benign condition, the association between migraine with aura and ischemic stroke is well established. ${ }^{2}$ According to the International Classification of Headache Disorders, the diagnosis of migrainous infarction can be made in the presence of a migraine attack, which occurs in patients fulfilling the criteria for migraine with aura, is typical of previous attacks except that one or more aura symptoms persist $>60$ minutes, and is associated with an ischemic brain lesion in a suitable territory as demonstrated by neuroimaging, when alternative diagnoses have been ruled out (https://www.ichd-3.org/). Migrainous infarction mostly involves the posterior circulation and occurs most commonly in women and patients younger than 45 years of age.

The exact physiopathology underpinning migrainous infarct has not been fully clarified. Changes in cerebral hemodynamic, increased vascular resistance, the breakdown of neuronal ion homeostasis, and release of neuroinflammatory mediators associated with cortical spreading depression are thought to play key roles. Endothelial dysfunction and

\author{
Mauro Silvestrini ${ }^{1}$
}

Address for correspondence Simona Lattanzi, MD, Department of Experimental and Clinical Medicine, Neurological Clinic, Marche Polytechnic University, Via Conca 71, 60020 Ancona, Italy (e-mail: alfierelattanzisimona@gmail.com).

impairment of endothelial repair capacity, reduced bioavailability of vasodilators and vasospasm, and hypercoagulable state induced by increased oxidative stress are also hypothesized to contribute. ${ }^{2}$ Independent risk factors of cerebral infarct in migraineurs include smoking, use of oral contraceptives, and migraine-specific medications such as triptans and ergot alkailoids ${ }^{2}$; the evidence about a possible link between migraine, patent foramen ovale, and stroke is still not conclusive. ${ }^{3}$

\section{Cortical Laminar Necrosis: From Pathophysiology to Clinic}

The CLN is a quite uncommon kind of cortical infarct characterized by the selective, delayed necrosis of the cerebral cortex, mainly of the third layer. The injury involves either neurons, glia, and blood vessels, and it is generally greater in the depths and sides of the sulci than over the crest of the gyri. ${ }^{4}$ It appears from 2 weeks to 2 years after brain infarct as pathognomonic high density on computed tomography scans and high-intensity signal on T1-weighted and fluid-attenuated inversion-recovery on magnetic resonance images, which show the curvilinear gyriform anatomy of the affected cerebral convolutions. ${ }^{4}$ The neuroimaging findings correspond to the neuronal damage, glial cell proliferation, accumulation of denatured proteins and deposition of fat-laden macrophages, and are not secondary to the hemorrhagic transformation of the infarct. ${ }^{4}$

The CLN generally results from critically long-lasting impairment of cerebral metabolism and energy production. The higher metabolic demand of the gray than white matter explains the greater vulnerability to energy failure and selective necrosis of the cortex. The CLN has been observed both in pediatric and adult age. The most common causes are hypoxic-ischemic encephalopathy and watershed infarction; other etiologies include hypoglycemia, status epilepticus, immunosuppressive and antineoplastic treatments, infections and autoimmune diseases of the central nervous system, moyamoya disease, migrainous infarction, metabolic disorders such as citrullinemia, and hyponatremia correction. ${ }^{4}$ 


\section{Predicting Outcome in Stroke Patients: The Multivariable Approach May Be the Answer}

The CLN is usually a clue for predicting poor neurological recovery, even when it occurs within topographically restricted brain lesions. Notwithstanding, complete regression of symptoms and deficits occurred in the reported case. On this ground, it is worth to notice that increasing evidence suggests that stroke recovery is multifactorial and depends on a huge number of variables linked to metabolism, immune reaction, inflammatory response, and cerebral perfusion..$^{5-9}$ These factors can interact to each other in additive or even synergistic fashion, and contribute to the degree of neurovascular recovery, development of secondary injury, and occurrence of systemic complications after stroke. Due to the great heterogeneity, complexity, and interdependence of the outcome modifiers, the reliable prediction of prognosis is a goal difficult to achieve through the traditional, currently available tools and models. Artificial intelligence may offer additional resources and is a candidate to change stroke medicine in the near future. Signally, as machine learning-based algorithms need aggregate millions of data to work efficiently, the systematic and structured collection of information within and across centers represents the conditio sine qua non to build "big" stroke databases and develop integrated models of care in the setting of the individualized medicine.

\section{Funding}

None.

\section{Conflict of Interest}

None declared.

\section{References}

1 Sharma SR, Massaraf H, Das S, Kalita A. Cortical laminar necrosis as a presenting manifestation of migraine in an apparently normal patient: a rare case report. J Neurosci Rural Pract 2019;10(3):559-562

2 Zhang Y, Parikh A, Qian S. Migraine and stroke. Stroke Vasc Neurol 2017;2(3):160-167

3 Lattanzi S, Brigo F, Cagnetti C, Di Napoli M, Silvestrini M. Patent foramen ovale and cryptogenic stroke or transient ischemic attack: To close or not to close? A systematic review and meta-analysis. Cerebrovasc Dis 2018;45(5)(6):193-203

4 Siskas N, Lefkopoulos A, Ioannidis I, Charitandi A, Dimitriadis AS. Cortical laminar necrosis in brain infarcts: serial MRI. Neuroradiology 2003;45(5):283-288

5 Lattanzi S, Bartolini M, Provinciali L, Silvestrini M. Glycosylated hemoglobin and functional outcome after acute ischemic stroke. J Stroke Cerebrovasc Dis 2016;25(7):1786-1791

6 Lattanzi S, Cagnetti C, Rinaldi C, Angelocola S, Provinciali L, Silvestrini M. Neutrophil-to-lymphocyte ratio improves outcome prediction of acute intracerebral hemorrhage. J Neurol Sci 2018;387:98-102

7 Zangari R, Zanier ER, Torgano G, et al; LEPAS group. Early ficolin-1 is a sensitive prognostic marker for functional outcome in ischemic stroke. J Neuroinflammation 2016;13:16

8 Lattanzi S, Cagnetti C, Pulcini A, et al. The P-wave terminal force in embolic strokes of undetermined source. J Neurol Sci 2017;375:175-178

9 Lattanzi S, Silvestrini M. Blood pressure in acute intra-cerebral hemorrhage. Ann Transl Med 2016;4(16):320 